\section{AUTORES}

Pedro Gabriel Silva Lemos ${ }^{1}$, Marco Antônio Moraes Duque ${ }^{1}$, Carla Nery Machado ${ }^{1}$

\section{Autor Correspondente: Carla} Nery Machado ${ }^{1}$

Carla-odonto543@gmail.com

\section{INSTITUIÇÃO AFILIADA}

1-Departamento de ciências odontológicas- Universidade Federal do Amazonas- Manaus, Amazonas, Brasil.

\section{CITAÇÃO}

LEMOS, Pedro Gabriel Silva, DUQUE, Antônio Moraes, MACHADO, Carla Nery. Componentes que afetam o medo no tratamento dentário em adultos: um estudo seccional. Brazilian Journal of Implantology and Health Sciences. v.1, n.4, p. 41-54, 2019.

\section{PALAVRAS CHAVE}

Medo, Ansiedade, Dentista, Escala

de Depressão

\section{TEMA: COMPONENTES QUE AFETAM O MEDO NO TRATAMENTO DENTÁRIO EM ADULTOS: UM ESTUDO SECCIONAL}

Introdução: A prevalência estimada de extremo medo e ansiedade dentária é de $40 \%$ na população adulta. 0 medo expresso pelo paciente do dentista raramente é usado na prática clínica para avaliar as preocupações do paciente.

Objetivos: O presente estudo foi realizado para identificar os fatores de risco associados ao medo do dentista e a frequência de visitas ao consultório odontológico.

Metodologia: O estudo incluiu 98 indivíduos que visitaram uma clínica odontológica da Universidade Federal do Amazonas. O medo dentário foi medido: (Qual o seu medo de visitar o dentista? A. De modo algum, b. Pouco c. Muito). Os dados demográficos foram coletados com base na Escala Hospitalar de Ansiedade e Depressão (HAD), enquanto o medo dentário foi avaliado pelo Questionário de Medo Dental (CMD). Essas avaliações foram feitas antes da realização do tratamento odontológico.

Resultados: Os resultados revelaram que quatro dimensões do TMC foram consideradas: A. cuidados acidentais, b. atitude do dentista, c. negligência e d. organização. Somente as dimensões de atitude do dentista $(O R=2,4$ (IC95\%: 1,1-5,4); $p=0,02$ ) e negligência (OR = 5,3 (IC95\%: 2,0-13,1); $p=$ 0,0001 ), juntamente com o os níveis de ansiedade (OR = 1,3 (IC95\%: 1,3-1,7); $p=0,01$ ) foram associados independentemente à presença de medo dentário. Nenhuma das variáveis esteve associada à frequência de visitas ao dentista.

Conclusões: Os resultados revelaram ainda que as dimensões de atitude e ansiedade do dentista estavam associadas ao medo de visitar o dentista. 


\title{
COMPONENTS AFFECTING DENTAL FEAR IN ADULTS: A SECTIONAL STUDY
}

\begin{abstract}
Introduction: The estimated prevalence of extreme dental fear and anxiety is $40 \%$ in the adult population. The patient's expressed fear of the dentist is rarely used in clinical practice to assess the patient's concerns.

Objectives: The present study was conducted to identify risk factors associated with fear of the dentist and the frequency of visits to the dental office.

Methodology: The study included 98 individuals who visited a dental clinic in Federal University of Amazonas. Dental fear was measured: (What is your fear of visiting the dentist? A. Not at all, b. Little c. Much). Demographic data were collected based on the Hospital Anxiety and Depression Scale (HAD), while dental fear was assessed by the Dental Fear Questionnaire (CMD). These evaluations were made prior to the dental treatment.
\end{abstract}

Results: The results revealed that four dimensions of CMD were considered: A. accidental care, b. attitude of the dentist, c. negligence and d. organization. Only the dimensions of dentist attitude $(\mathrm{OR}=2.4(95 \% \mathrm{Cl}: 1.1-5.4) ; \mathrm{p}=0.02)$ and negligence $(\mathrm{OR}=5.3(95 \% \mathrm{Cl}: 2.0-13, p=0.02) ; 1) ; p=0.0001)$, along with anxiety levels $(\mathrm{OR}=1.3(95 \% \mathrm{Cl}: 1.3-1.7) ; \mathrm{p}=0.01)$ were independently associated with the presence of dental fear. None of the variables was associated with the frequency of dental visits.

Conclusions: The results also revealed that the attitude and anxiety dimensions of the dentist were associated with the fear of visiting the dentist.

KEY WORD: Fear, Anxiety, Dentist, Depression Scale

Pedro Gabriel Silva Lemos - Undergraduate Student of Dentistry at UFAM

Marco Antônio Moraes Duque - Student of Dentistry at UFAM

Carla Nery Machado - PhD in Dentistry and Prof. of Undergraduate Dentistry at UFAM 


\section{INTRODUÇÃO}

Lidar com o medo do consultório odontológico é vivenciado diariamente pelos profissionais de saúde bucal. Até $40 \%$ de todos os indivíduos relatam ter medo do dentista [1]. Por sua vez, 3-5\% da população tem fobia extrema de dentistas [2]. Essa fobia pode se manifestar de muitas maneiras diferentes, incluindo evitar o consultório odontológico, que por sua vez pode resultar em negligência na assistência à saúde bucal. Além disso, a conscientização da falta de saúde bucal pessoal e as emoções de vergonha perpetuam a prevenção do dentista. Isso foi descrito como um ciclo vicioso que explica o comportamento de esquiva [3]. O medo dentário extremo também está associado a diferentes tipos de tratamento dentário, secundários ao atendimento diferido. Nesses casos, tratamentos menos conservadores não estão disponíveis [4].

Embora esses pacientes experimentem um medo intenso, eles se sujeitam a estímulos assustadores, embora com mais consultas canceladas do que pacientes sem fobia. Esses indivíduos que sofriam de extremo medo dentário sentiram dor e sofreram trauma quando a anestesia foi aplicada. Estudos mostraram que o paciente administrado com terapias dentárias, como perfuração, extração, injeção, enquanto outros relatam medo de instrumentos como agulhas ou exploradores. Outros atribuem seu medo do consultório odontológico ao fato de serem colocados em uma situação em que não têm controle e são vistos com menosprezo [5].

Lidar com esses indivíduos é importante para melhorar os cuidados de saúde bucal. Um estudo realizado por Vassend [6], revelou que pacientes com medo intenso têm um número significativamente maior de dentes perdidos, mais superfícies dentárias deterioradas, mais lesões peri-radiculares e maior perda óssea quando comparados àqueles sem medo dentário. Outro estudo publicado por Kent [7], demonstrou que esse grupo de pessoas considerava esse medo reduzir significativamente sua qualidade de vida. Berggren [8], 
relatou que houve um aumento na dosagem dos medicamentos, mais dias de trabalho perdidos e relacionamentos ruins entre os indivíduos.

\section{Objetivos do estudo}

O presente estudo considera três objetivos:

- Descrever os medos dentários expressos pelos pacientes que visitam o consultório odontológico

- Identificar fatores que fazem parte do conceito de medo dental

- Identificar fatores que distinguem pacientes que têm extremo medo dental dos pacientes que não têm medo dental.

\section{METODOLOGIA}

Foi realizado um estudo clínico transversal para descobrir os componentes que afetam o medo dentário em adultos: um estudo transversal, envolvendo a administração das escalas Hospitalar de Ansiedade e Depressão (HAD) [9] e questionário sobre o medo da dentista (Cuestionario de Miedos Dentales (CMD) [10]. Este estudo foi realizado na clínica odontológica da Universidade federal do Amazonas, durante um período de 13 meses e teve seu modelo aprovado pelo comitê de ética sob o número 2.431.331.

\section{0 critério}

Os critérios desta investigação foram pacientes que falam espanhol, com idades entre 18 e 65 anos, assinaram o termo de consentimento e devem responder aos questionários da escala de ansiedade e depressão (Hospital Anxiety and Depression Scale, HAD). Os critérios excluídos neste estudo foram uso de medicamentos psicotrópicos (antidepressivos, benzodiazepínicos, antipsicóticos) e pacientes diagnosticados com dependência de álcool ou drogas. 


\section{Os entrevistados}

Os entrevistados foram compostos por 98 pacientes com idade média de 37 anos. O pesquisador explicou os objetivos do estudo aos participantes pretendidos.

\section{Procedimentos}

Os dados obtidos neste estudo foram analisados no Statistical Package for the Social Sciences (SPSS versão 12). As associações foram testadas usando análises de regressão linear descritiva, univariada e multivariada. A significância estatística foi considerada para o p1, com rotação varimax. A comparação dos sujeitos com medo baixo versus alto foi baseada no teste do qui-quadrado e no teste $t$ de Student. Em seguida, aplicamos uma análise multivariada usando regressão logística. As variáveis dependentes foram o medo do dentista (baixo / alto) e a frequência das visitas (regulares / não regulares ou nunca), enquanto as variáveis independentes foram sexo, idade e as dimensões extraídas da análise do componente principal que se mostraram significativas no estudo univariado análise.

\section{RESULTADOS E DISCUSSÃO}

\section{Dados sociodemográficos}

O estudo incluiu 98 indivíduos que visitaram consecutivamente a clínica odontológica da Universidade federal do Amazonas. Quatro pacientes se recusaram a responder às perguntas do estudo e dois tiveram que ser retirados devido a erros no atendimento das escalas. Cinquenta e nove dos sujeitos eram mulheres (60\%). A média de idade \pm desvio padrão (DP) foi de $37,5 \pm 12,6$ anos (tabela 1). Quarenta e nove dos pacientes entrevistados (50\%) visitavam o dentista regularmente, enquanto 43 somente 0 
experimentavam quando experimentavam desconforto (44\%) e 6 alegavam nunca visitar o dentista (6\%). Quanto ao medo de visitar a clínica odontológica, $81 \%$ dos pacientes $(n=79)$ relataram baixo medo: $45 \%(n=44)$ não relataram medo, enquanto $36 \%(n=35)$ admitiram sentir algum medo. Por sua vez, 19 pacientes (19\%) relataram alto medo do dentista. Os escores médios \pm desvio padrão (DP) nas sub escalas de ansiedade e depressão do HAD foram de 3,1 $\pm 2,4$ e $7,3 \pm 3,2$, respectivamente (Tabela 1 ).

Fatores extraídos da análise de componentes principais do questionário de medo dental (TMC). Após a análise dos componentes principais das dimensões da TMC com rotação varimax, extraímos quatro fatores com um autovalor> 1 (Tabela 2): "Assistência odontológica", contabilidade por 25,8\% da variância; "Atitude do dentista", representando $15,7 \%$ da variância; "Negligência", respondendo por 13,8\% da variação; e "organização", representando $13,2 \%$ da variação. O primeiro fator ("atendimento odontológico") incluiu os seguintes itens: O trabalho é realizado pelos auxiliares, ferindo meus lábios com algum instrumento, brusquidão por parte do dentista, auxiliares desagradáveis, atendimento de muitos pacientes ao mesmo tempo, dentista mal-educado, dentista com mau hálito, ferindo minha boca, falta de gentileza no tratamento.

Tabela 1. Fatores sociodemográficos e escores hospitalares de ansiedade e depressão

\begin{tabular}{lc}
\hline & $\mathrm{N}(\%)$ \\
\hline Participantes (mulheres) & $59(60 \%)$ \\
Frequência de visitas ao dentista & $49(50 \%)$ \\
Regularmente & $43(44 \%)$ \\
Somente em caso de desconforto & $6(6 \%)$ \\
Nunca & \\
Medo do dentista & $79(81 \%)$ \\
Baixo medo & $44(45 \%)$ \\
Nada & $35(36 \%)$ \\
Um pouco de & \\
Alto medo & $19(19 \%)$ \\
Muito & $37,5(12,6)$ \\
Média (DP) & $3,1(2,4)$ \\
Idade (anos) & $7,3(3,2)$ \\
HAD-ansiedade & \\
HAD-depressão &
\end{tabular}


Tabela 2. Fatores extraídos da análise de componentes principais

\begin{tabular}{|c|c|c|c|c|}
\hline & $\begin{array}{l}\text { Fator "Atendimento } \\
\text { odontológico" }\end{array}$ & $\begin{array}{l}\text { "fator de atitude } \\
\text { do dentista" }\end{array}$ & $\begin{array}{l}\text { fator } \\
\text { "negligência" }\end{array}$ & $\begin{array}{l}\text { Fator } \\
\text { "organização" }\end{array}$ \\
\hline $\begin{array}{l}\text { O trabalho é realizado } \\
\text { pelos assistentes }\end{array}$ & 0,81 & & & \\
\hline $\begin{array}{l}\text { Ferindo meus lábios } \\
\text { com algum instrumento }\end{array}$ & 0,79 & & & \\
\hline $\begin{array}{l}\text { Brusquidão por parte do } \\
\text { dentista }\end{array}$ & 0,72 & & & \\
\hline Assistentes desagradáveis & 0,69 & & & \\
\hline $\begin{array}{l}\text { O atendimento de muitos pacientes } \\
\text { ao mesmo tempo }\end{array}$ & 0,67 & & & \\
\hline Dentista indelicado & 0,67 & & & \\
\hline Dentista com mau hálito & 0,58 & & & \\
\hline Ferindo minha boca & 0,56 & & & \\
\hline $\begin{array}{l}\text { Falta de gentileza no } \\
\text { Tratamento }\end{array}$ & 0,56 & & & \\
\hline Reprimendas & & 0,85 & & \\
\hline $\begin{array}{l}\text { Mau humor por parte do } \\
\text { Dentista }\end{array}$ & & 0,75 & & \\
\hline Injeção nas gengivas & & & 0,76 & \\
\hline Sangramento na boca & & & 0,72 & \\
\hline Uso do afastador oral & & & 0,64 & \\
\hline Erros na colocação da injeção & & & 0,59 & \\
\hline $\begin{array}{l}\text { Revistas antigas na sala de } \\
\text { espera }\end{array}$ & & & 0.51 & \\
\hline Atrasos no atendimento de & & & & 0,81 \\
\hline $\begin{array}{l}\text { Distribuição da clínica } \\
\text { Odontológica } \\
\text { Variacão percentual cumulativa }\end{array}$ & $25,8 \%$ & $41,5 \%$ & $55,3 \%$ & $\begin{array}{l}0,74 \\
68,5 \%\end{array}$ \\
\hline
\end{tabular}

O segundo fator ("atitude do dentista") incluiu os seguintes itens: Repreensões e mau humor por parte do dentista. O terceiro fator ("negligência") incluiu os seguintes itens: injeção na gengiva, sangramento na boca, uso do afastador oral, erros na colocação da injeção. Por fim, o quarto fator ("organização"), relacionado às questões de gestão na clínica 
odontológica, incluía os seguintes itens: Atrasos no atendimento e distribuição da clínica odontológica.

\section{Fatores Associados ao Medo}

\section{Análise Univariada do Dentista}

$\mathrm{Na}$ análise univariada, o escore da sub escala de ansiedade do HAD (HAD-A), juntamente com os fatores "negligência" e "atitude do dentista" (este último no limite de significância), foram associados ao medo percebido pelo paciente de visitar o dentista.

Aqueles com alto medo apresentaram maior pontuação no HAD-A do que aqueles com baixo medo $(9,2 \pm 3,2$ vs. $6,8 \pm 3,0 ; p<0,005)$. Da mesma forma, aqueles com alto medo do dentista relataram desconforto aumentado em comparação com aqueles com baixo medo, nos itens relacionados aos fatores "negligência" $(0,79 \pm 0,70$ vs. $-0,19 \pm 0,96 ; p<0,0001)$ e "atitude do dentista " $(0,35 \pm 0,9$ vs. vs. $-0,84 \pm 1,0 ; p<0,07)$.

Outros fatores como idade, sexo e depressão sintomas (escore HAD-D) não foram associados ao paciente medo do dentista, da mesma maneira que os "fatores cuidados "e" organização " (Tabela 3).

\section{Análise multivariada}

$\mathrm{Na}$ análise multivariada, os fatores "negligência" e "atitude do dentista", além de HAD-A, idade e sexo foram inseridos como variáveis independentes. As três variáveis consideradas associadas independentemente ao medo de dentistas eram HAD-A ( $O R=1,3(1,0-1,7) ; p<0,01)$, o fator negligência (OR $=5,3(2,0-13,7) ; p<0,0001)$ e o fator "atitude do dentista" $(O R=2,4(1,1-5,4)$; $\mathrm{p}<0,0001$ ) (tabela 4).

\section{Fatores Associados à Frequência de Visitas ao Dentista}

Como mostra a Tabela 5, nenhum dos fatores estudados foi constatou estar associado à frequência de visitas ao Clínica odontológica. 


\section{Discussão}

O principal achado deste estudo é que os fatores "Negligência" e "atitude do dentista" são independentemente associada ao alto medo do dentista.

No presente estudo, o medo do dentista esteve associado para altos escores de ansiedade. Da mesma forma, a ansiedade dental está relacionada à condição geral de ansiedade do paciente [11]. Isso poderia sugerir que o medo de visitar o dentista pode ser incluído entre as várias fobias. Consequentemente, pode-se deduzir que intervenções que demonstraram ser eficazes em tratamento de fobias específicas também podem ser aplicadas a pacientes com medo do dentista.

O uso de tais técnicas seria proporcionar melhorias no tratamento odontológico, resultando em menor tempo de espera da anestesia, necessidade de menores quantidades anestésico, menos visitas canceladas e mais frequentes visitas à clínica odontológica [12].

A ansiedade dental tem sido relacionada à deficiência bucal saúde em crianças e adultos. Os autores $[13,14,15,16]$ concluíram que a prevenção de tratamento odontológico está relacionada a altos escores de ansiedade e alto índice de CPOD (dentes cariados, ausentes, cheios) pontuações. Consequentemente, esses pacientes apresentam maior risco de visitas irregulares e / ou cancelamento de visitas à clínica odontológica $[17,18]$.

Table 3. Univariate analysis of factors associated to fear of the dentist

\begin{tabular}{lccc}
\hline & $\begin{array}{c}\text { Baixo medo do dentista } \\
\text { Media } \pm \text { SD }\end{array}$ & $\begin{array}{c}\text { Alto medo do dentista } \\
\text { Media } \pm S D\end{array}$ & Valor de P \\
\hline Idade (anos, \pm DP) & $36,9 \pm 12,9$ & $40,2 \pm 11,2$ & 0,31 \\
Fatores & $-0,72 \pm 1,0$ & $0,30 \pm 0,81$ & 0,14 \\
"Atendimento odontológico" & $-0,84 \pm 1,0$ & $0,35 \pm 0,9$ & $0,07^{*}$ \\
"Atitude do dentista" & $-0,19 \pm 0,96$ & $0,79 \pm 0,70$ & $0,0001^{* *}$ \\
"Negligência" & $-0,00001 \pm 0,99$ & $0,0003 \pm 1,0$ & 0,99 \\
"Organização" & $6,8 \pm 3,0$ & $9,2 \pm 3,2$ & $0,005^{* *}$ \\
Ansiedade HAD & $3,2 \pm 2,5$ & $2,5 \pm 1,9$ & 0,26 \\
Depressão HAD & $\mathrm{N}(\%)$ & $\mathrm{N}(\%)$ & \\
& $47(59,5 \%)$ & $12(63 \%)$ & 0,77 \\
Sexo (mulheres,\%) & & $11(57 \%)$ & 0,44 \\
\hline
\end{tabular}


Tabela 4. Análise de regressão logística do medo dental percebido (alto versus baixo)

\begin{tabular}{lcccc}
\hline & $\mathrm{B}$ & Wald $(\mathrm{df}=1)$ & OR $(\mathrm{IC} 95 \%)$ & Valor P \\
\hline HAD-A & 0,320 & $6-4$ & $1,3(1,0-1,7)$ & 0,010 \\
"Atitude do dentista" & 0,900 & 5,000 & $2,4(1,1-5,4)$ & \\
& 0,020 & & & \\
"Negligência" & 1.600 & 12.200 & $5,3(2,0-13,7)$ & 0,000 \\
Idade & $-0,002$ & 0,008 & $0,99(0,93-1,0)$ & 0,920 \\
Sexo & 0,800 & 1,300 & $2,2(0,56-8,7)$ & 0,250 \\
\hline
\end{tabular}

Tabela 5. Fatores associados à frequência de visitas ao dentista

\begin{tabular}{llcr}
\hline Fator & $\begin{array}{c}\text { Visitas regulares } \\
(\text { Média } \pm \mathrm{DP})(\mathrm{n}=49)\end{array}$ & $\begin{array}{c}\text { Não há visitas regulares } \\
(\text { Média } \pm \mathrm{DP})(\mathrm{n}=49)\end{array}$ & Valor $\mathrm{P}$ \\
\hline "Atendimento odontológico" & $0,005 \pm 1,0$ & $-0,005 \pm 1,0$ & 0,95 \\
"Atitude do dentista" & $-0,07 \pm 1,1$ & $0,07 \pm 0,88$ & 0,46 \\
"Negligência" & $-0,10 \pm 0,98$ & $0,10 \pm 1,0$ & 0,32 \\
"Organização" & $0,12 \pm 1,0$ & $-0,12 \pm 0,92$ & 0,23 \\
HAD-A & $7,1 \pm 2,7$ & $7,5 \pm 3,6$ & 0,49 \\
HAD-D & $2,8 \pm 2,3$ & $3,4 \pm 2,5$ & 0,23 \\
\hline
\end{tabular}

Vários estudos mostraram que pacientes com ansiedade precisam de mais tempo para serem atendidos, o que implica um custo adicional para o profissional de odontologia. Além disso, pacientes desse tipo têm menor probabilidade de ficarem satisfeitos com o tratamento recebido; portanto, seu cuidado adequado pode representar um desafio para o dentista $[18,19]$. Os níveis de ansiedade e depressão foram semelhantes aos relatados em uma grande amostra de pacientes com dor nas costas aguda [20].

Fatores como a interação dentista-paciente são cruciais para o controle da ansiedade. Nesse contexto, a atitude e os comentários do dentista foram identificados como fatores muito importantes em nosso estudo. Moore [21], encontraram contatos negativos com o dentista para fazer com que os pacientes identificassem a origem de sua ansiedade no consultório odontológico. Nesse sentido, identificamos a "atitude do dentista" como um fator independente associado ao alto medo do dentista. Pacientes com ansiedade importante tendem a ficar excessivamente cansados após uma consulta odontológica. 
A ansiedade intensa pode ter efeitos cognitivos importantes nos pacientes, com pensamentos negativos, medo, choro, agressividade, distúrbios do sono e alterações nos hábitos alimentares, aumento da automedicação e falta de confiança pessoal [22]. Verificou-se que as técnicas de relaxamento e distração são eficazes para diminuir a ansiedade entre os pacientes que visitam a clínica odontológica [23]. Algumas técnicas de distração na prática odontológica são aromaterapia [13], musicoterapia [17], visualização de imagens ou imagens relaxantes [6] e uso de técnicas de realidade virtual com óculos 3D [17]. Outras técnicas para diminuir a ansiedade incluem a técnica de relaxamento muscular progressivo desenvolvida por Jacobsen, que induz o relaxamento através da redução da tensão muscular, contraindo e relaxando os músculos por grupos [11]. Outro método simples para produzir relaxamento compreende exercícios de respiração rítmica, nos quais o paciente inspira profundamente, prendendo a respiração por 5 segundos e depois expirando por outro intervalo de 5 segundos [1]

A hipnose é considerada uma ferramenta importante para superar 0 medo dentário. Os resultados confirmam que a hipnose é benéfica como uma intervenção adjunta para reduzir a ansiedade em pacientes, principalmente em vista de sua natureza não invasiva. Algumas descobertas mostram que a hipnose não é apenas benéfica, mas também é muito facilmente aceita pelos pacientes. A implementação da hipnose no atendimento odontológico de rotina deve ser incentivada [15].

A administração de relaxantes como benzodiazepínicos por diferentes vias (oral, retal, intranasal intravenosa) é eficaz e é comumente prescrita para prevenir a ansiedade na prática odontológica.

Sua combinação com sedação (óxido nitroso e oxigênio) é eficaz e constitui uma alternativa à anestesia geral [19]. Esses medicamentos agem rápida e brevemente, a condição do paciente retornará ao seu estado normal em um curto período de tempo [17]. 
Dois fatores foram relacionados ao alto medo do dentista em nosso estudo: a "atitude do dentista" e a preocupação com a "negligência". Nesse contexto, a comunicação paciente-dentista desempenha um papel crucial. $\mathrm{O}$ apoio verbal e a segurança são estratégias comumente usadas e, além disso, devem ser praticadas por todos os membros da equipe odontológica. Consequentemente, as consultas odontológicas envolvendo pacientes particularmente ansiosos devem ter uma duração mais longa, a fim de permitir que o dentista explique completamente o procedimento e forneça o tratamento necessário [20].

Neste estudo, a preocupação com a negligência foi um dos fatores associados ao medo do dentista. Vários autores tentaram diminuir a ansiedade por meio de diferentes técnicas, como intervenções psicoeducacionais que se mostraram eficazes em outras disciplinas [22]

Assim, estão disponíveis intervenções simples que podem ser aplicadas pelo dentista e membros da equipe na clínica odontológica. Para isso, primeiro precisamos detectar os pacientes com ansiedade importante e avaliar o procedimento mais adequado a cada caso individual. A inclusão de conceitos comportamentais e medidas de educação odontológica nos programas de treinamento odontológico poderia melhorar a capacidade dos futuros profissionais de odontologia de lidar adequadamente com pacientes que sofrem altos níveis de ansiedade [2]

\section{CONCLUSÃO}

A ansiedade e o medo do dentista são fenômenos multifatoriais e comuns na população em geral. Os profissionais de odontologia devem ser capazes de identificar pacientes com ansiedade importante e devem estar familiarizados com os procedimentos aplicáveis antes do tratamento odontológico, a fim de garantir um gerenciamento bem-sucedido do paciente. Os fatores "atitude do dentista" e "negligência", juntamente com a ansiedade, foram identificados como parâmetros independentes associados ao alto medo do tratamento odontológico. 


\section{REFERENCES}

1- Abrahamsson, K.H., U. Berggren and S.G. Carlsson, 2000. Psychosocial aspects of dental and general fears in dental phobic patients. Acta Odontol. Scand., 58: 37-43. DOI: 10.1080/000163500429415

2- Abrahamsson, K.H., U. Berggren, M. Hakeberg and S.G. Carlsson, 2001. Phobic avoidance and regular dental care in fearful dental patients: A comparative study. Acta Odontol. Scand., 59: 273-279. DOI: 10.1080/000163501750541129

3- Aitken, J.C., S. Wilson, D. Coury, A.M. Moursi, 2002. The effect of music distraction on pain, anxiety and behavior in pediatric dental patients. Pediatr. Dent., 24: 114-118. PMID: 11991313

4- Armfield, J.M., G.D. Slade and A.J. Spencer, 2009. Dental fear and adult oral health in Australia. Community Dent. Oral Epidemiol., 37: 220-230. DOI: 10.1111/j.1600-0528.2009.00468.x

5- Berggren, U., 2002. Long-term management of the fearful adult patient using behavior modification and other modalities. J. Dent. Educ., 65: 1357-1368. PMID: 11780654

6- Busch, V., W. Magerl, U. Kern, J. Haas and G. Hajak et al., 2012. The effect of deep and slow breathing on pain perception, autonomic activity and mood processing- an experimental study. Pain Med., 13: 215-228. DOI: 10.1111/j.1526-4637.2011.01243.x

7- PARAGUASSU, Éber Coelho; LACERDA, Jamille dos Passos. Oral health of the elderly in Brazil: Systematic review. Brazilian Journal of Implantology and Health Sciences, v.1, n.2, p. 25-33, 2019.

8- Cohen, S.M., J. Fiske and J.T. Newton, 2000. The impact of dental anxiety ondaily living. Br. Dent. J., 189: 385-390. PMID: 11081950

9- Corah, N.L., E.N. Gale and S.J. Illig, 1979. The use of relaxation and distraction to reduce psychological stress during dental procedures. J. Am. Dent. Assoc., 98: 390-394. DOI: 10.14219/jada.archive.1979.0049

10- Coulthard, P. and D. Craig, 1997. Conscious sedation. Dent. Update, 24: 376-381. Economou, G.C., 2003. Dental anxiety and personality: Investigating the relationship between dental anxiety and selfconsciousness. J. Dent. Educ., 67: 970-980. PMID: 14518835

11- Eitner, S., M. Wichmann, A. Paulsen and S. Holst, 2006. Dental anxiety- an epidemiological study on its clinical correlation and effects on oral health. J. Oral. Rehabil., 33: 588-593. DOI: 10.1111/j.13652842.2005.01589.x

12- Fox, C. and J.T. Newton, 2006. A controlled trial of the impact of exposure to positive images of dentistry on anticipatory dental fear in children. Community Dent. Oral. Epidemiol., 34: 455-459. DOI: 10.1111/j.1600-0528.2006.00303.x 
13-Glaesmer, H., H. Geupel and R. Haak, 2015. A controlled trial on the effect of hypnosis on dental anxiety in tooth removal patients. Patient Educ. Couns., 98: 1112-1115. DOI: 10.1016/j.pec.2015.05.007

14- Hainswoth, J.M., H. Moss and K.J. Fairbrother, 2005. Relaxation and complementary therapies: An alternative approach to managing dental anxiety in clinical practice. Dent. Update, 32: 90-92. PMID: 15819152

15-Hmud, R. and L.J. Walsh, 2009. Ansiedad dental: Causas, complicaciones y métodos de manejo. J. Minim. Interv. Dent., 2: 237248.

16-Jafarzadeh, M., S. Arman and F.F. Pour, 1985. Effect of aromatherapy with orange essential oil on salivary cortisol and pulse rate in children during dental treatment: A randomized controlled clinical trial. Adv. Biomed. Res., 2: 10-10. DOI: 10.4103/2277-9175.107968

17- Kent, G., 1985. Cognitive processes in dental anxiety. British J. Clin. Psychol., 24: 259-264. DOI: 10.1111/j.2044-8260.1985.tb00658.x

18-Kohl, F., 2002. Progressive muscle relaxation according to $\mathrm{E}$. Jacobson. A modern relaxation technique. Med. Monatsschr. Pharm., 25: 77-87. PMID: 11925766

19-Lehrner, J., C. Eckersberger, P. Walla, G. Pötsch and L. Deecke, 2000. Ambient odor of orange in a dental office reduces anxiety and improves mood in female patients. Physiol. Behav., 71: 83-86. DOI: 10.1016/S0031-9384(00)00308-5

20-PARAGUASSU, Éber Coelho et al. Qualidade de vida e satisfação em usuários de prótese total no estado do Amapá, Brasil. Revista Eletrônica Acervo Saúde, n. 27, p. e876-e876, 2019

21-Murthy, A.K., M. Pramila and S. Ranganath, 2014. Prevalence of clinical consequences of untreated dental caries and its relation to dental fear among 12-15-year-old schoolchildren in Bangalore city, India. Eur. Arch. Paediatr. Dent., 15: 45-49. DOI: 10.1007/s40368-013-0064-1

22-Navarro, C. and R. Ramírez, 1996. Un estudio epidemiológico acerca de la prevalencia de ansiedad y miedos dentales entre la población adulta de la gran área metropolitana de Costa Rica. Psicol. Conductual, 4: 79-95.

23-Pohjola, V., A.K. Mattila, M. Joukamaa and S. Lahti, 2011. Anxiety and depressive disorders and dental fear among adults in Finland. Eur. J. Oral Sci., 119: 55-60. DOI: 10.1111/j.1600-0722.2010.00795.x 